\title{
Infectious Diseases of North American Black Bears (Ursus americanus): Viral and Bacterial Pathogens (Part One)
}

\author{
Mark A. Mitchell, DVM, MS, PhD; ${ }^{1}$ Dawn Zimmerman, DVM, MS ${ }^{2}$ \\ ${ }^{1}$ Louisiana State University, Baton Rouge, Lousiana; ${ }^{2}$ Memphis Zoo, Memphis, Tennessee
}

\begin{abstract}
Black bears (Ursus americanus) serve as an important keystone species within an ecosystem, and can also serve as sentinels for environmental health. Wildlife rehabilitators, biologists, and veterinarians working with these animals should familiarize themselves with infectious diseases commonly associated with these ursids. The purpose of this article is to provide a literature review of the common viral and bacterial pathogens associated with black bears.
\end{abstract}

\section{INTRODUCTION}

The American black bear (Ursus americanus), formerly widespread throughout North America, is now found only in scattered, remnant populations. Current estimates suggest that there are as few as 330,000 bears in the United States. In the southeastern United States, black bear populations are extremely low, being limited to rugged mountainous terrain and coastal plain swamps. The primary reasons for black bear declines are illegal poaching and habitat loss or degradation.

The black bear is an important sentinel species for its ecosystem because it is omnivorous, solitary in nature, long-lived (averaging 15 years in the wild), lacks natural predators (excluding man), and maintains a large home range. Therefore, health surveys of black bear populations can provide valuable information about exposure to infectious or parasitic agents in that region. This information can be used by wildlife biologists to make informed decisions regarding the conservation of this important species and provide insight to public health officials regarding zoonotic diseases within a specified area.

The purpose of this article is to provide a review

Dr. Mitchell is a former member of the NWRA Board of Directors. He is Assistant Professor of Zoological Medicine, and Director of the Wildlife Hospital of Louisiana at the Louisiana State University in Baton Rouge, LA.

Dr. Zimmerman graduated from Ross University after a clinical year at Louisiana State University. She completed an internship in zoological medicine at Omaha's Henry Doorly Zoo, and is currently the associate veterinarian at Memphis Zoo. of the literature pertaining to infectious agents observed in black bears in North America. Numerous reports on viral and bacterial agents known to infect the black bear were reviewed and grouped by agent, location/year, and prevalence. When potentially significant, correlations to age, sex, and season, as well as relationships to other species and pathogenicity of the agent, are discussed.

This infectious disease information is useful to wildlife rehabilitators, biologists, and veterinarians studying the black bear and managing the health of individual bears, wild populations, and their associated ecosystem. From baseline data, researchers can follow the increase or decrease in prevalence or incidence of infectious agents over time and note the season or location in which they are predominantly found. It is also important to acknowledge any clinical significance associated with infections and any public health threat of zoonotic diseases to wildlife rehabilitators, hunters, and researchers.

The 95 percent binomial confidence intervals (CI) were calculated for each of the prevalence estimates (Hassard 1991). In cases where the prevalence estimate was zero, the 95 percent confidence intervals were calculated with the technique described by van Belle and Millard (1998). The 95 percent confidence interval is used to acknowledge with 95 percent confidence that the population mean is within the interval. This is an important statistic to calculate because small study populations can provide large confidence intervals that diminish our ability to discern a population prevalence with much confidence. The reader should note that the size of a 95 percent $\mathrm{CI}$ is inversely proportional to the sample size.

\section{Viral Agents}

Bluetongue virus (BTV), also known as epizootic hemorrhagic disease, is a potentially devastating disease of 
ruminants from sub-tropical and tropical areas. This virus is primarily spread via arthropods. Clinically affected ruminants may develop epistaxis (nose-bleeding), diarrhea, and hoof problems. Mortalities can be significant in naïve populations. Although not considered an important disease in omnivores and carnivores, exposure to BTV has been reported in black bears from Florida (Dunbar et al 1998). Of the 61 bears sampled, three $(5 \%, 95 \% \mathrm{CI}: 0-10)$ were seropositive for BTV. The 95 percent CI suggest that exposure to BTV in Florida bears may not be as rare as expected, with as many as 10 percent of all bears potentially being exposed. The effect of BTV on ursids is unknown. However, experimental infections of domestic dogs with BTV can cause abortions and death (Dunbar et al 1998). Because bears are phylogenetically related to canids, similar clinical signs might occur in ursids. With the reduction of habitat and increased likelihood for contact between bears and other wildlife, such as cervids, the likelihood for exposure to BTV for ursids may increase.

Because of the genetic relationship between canids and ursids, it is not surprising that bears are susceptible to other viral agents known to affect dogs. Among these are canine adenovirus type one, canine distemper virus, and canine parvovirus. Exposure to canine adenovirus type one (CAV-1), the agent of infectious canine hepatitis, has been reported in black bears from Georgia, South Dakota, and Florida. In Georgia (Pursell et al 1983), CAV-1 was isolated from two captive cubs with clinical and postmortem signs consistent with infectious canine hepatitis. The affected bear cubs were ataxic, produced excess saliva, vomited, convulsed, and had nystagmus. The source of the infection was unknown, but it was speculated that the handlers might have served as the point source of infection. Because of this potential risk, some biologists suggest vaccinating captive bears prophylactically with a killed vaccine. Unfortunately, the efficacy and potential for side effects associated with CAV-1 vaccination in bears is unknown. In a South Dakota park, 19 percent ( $n=28,95 \%$ CI: $13-25$ ) of 148 black bears were seropositive for CAV-1 (Collins et al 1984). Most of the affected bears died within 12 hours of clinical signs. At first, wolves in the park vaccinated for CAV-1 were considered the source of infection for the bears. However, the virus isolated from the bears proved virulent to dogs and therefore was not considered to be of vaccine origin (Whetstone et al 1988). Canine adenovirus type one infected black bears have also been discovered in Florida (6\%, 95\% CI: 0-12), where the virus is believed to be transmitted directly or indirectly from wild canids, skunks, and domestic dogs (Dunbar et al 1998). Young bears appear more susceptible to the virus because of decreased immunotolerance. Because bears and canids coexist in many ecosystems, CAV-1 infection may be more prevalent than indicated and should be considered as a possible cause of mortality in free-ranging black bears (Pursell et al 1983).

Canine distemper virus (CDV) is a significant disease of wild canids, mustelids, and procyonids. Affected animals may present with diarrhea, lethargy, coughing, dyspnea, seizures, and/or severe neurological disease. Historically, bears were not considered to be susceptible to CDV; however, limited research has evaluated CDV exposure or infection in black bear populations. Dunbar et al (1998) identified serologic titers to CDV in eight percent (95\% CI: 2-14) of the 66 Florida bears they sampled. Whether CDV causes clinical disease in black bears is unknown. Wildlife rehabilitators, biologists, and veterinarians working with bears should consider CDV in differential diagnoses for animals presenting with gastrointestinal, respiratory, and neurological disease. In addition to CDV seropositive bears, Dunbar et al (1998) also identified 10 (16\%, 95\% CI: 7-25) bears that were seropositive for canine parvovirus (CPV). Neither CDV nor CPV has been isolated from black bears; therefore, it is impossible to determine whether these bears were infected or simply exposed to the viruses. Exposure to CDV and CPV may possibly occur in black bears, as with CAV-1, because ursids share habitat with canids and procyonids that harbor these viruses.

Rabies virus is an important zoonotic pathogen. However, reports of rabies in black bears are rare. Over a 40-year period (1956 to 1996) in Ontario, Canada, only seven confirmed cases of rabies were reported in black bears (Walroth et al 1996). The arctic fox strain was identified in all seven, indicating possible transmission by rabid foxes. Since 1977, only six human fatalities in Ontario were due to black bear attacks and all were rabies negative (Walroth et al 1996). In a second study, however, Wandeler and Casey (1993) confirmed rabies in 22 percent (two of nine) of the black bears tested from eastern Canada. Rabies may be more prevalent than previously thought, as rabid bears likely go unnoticed unless they are aggressive and encroach on human populations (Walroth et al 1996).

Pseudorabies virus (PrV) is a significant disease in swine, and has been associated with significant monetary losses in swine operations. Swine are the definitive host for $\operatorname{PrV}$, while wildlife are accidental hosts. Exposure to $\mathrm{PrV}$ in wild bears has only been 
reported in Florida. This was not unexpected, as Florida is known to have the largest feral swine population in the United States (Pirtle et al 1986). Florida black bears readily utilize feral swine as food, and 35 percent of the feral swine population in Florida was seropositive for PrV in 1993 (van der Leek et al 1993). Pirtle et al (1986) found that eight percent (95\% CI: 0-22) of the Florida black bears tested were seropositive for PrV. In another study from Florida, Dunbar et al (1998) found that none (0\%, 95\% CI: 0-8) of the 37 black bears they tested were seropositive. The results were not unexpected as the authors considered that there was a low probability of exposure and/or a high mortality rate following exposure (Dunbar et al 1998). Species other than swine are considered deadend hosts, and it is assumed that the mortality rate for dead-end hosts approximates 100 percent. There has been one confirmed black bear death associated with PrV (Schultze et al 1986). The bear had been raised on a PrV positive swine farm in Michigan. The presenting clinical signs in the bear included depression, fever, excess salivation, and convulsions. Individuals working with black bears in PrV endemic areas should consider this disease in a differential list when a bear presents with fever and neurological disease.

Arboviruses have become a significant health concern for both humans and wildlife in North America. Although several groups of these viruses, including Eastern equine encephalitis (EEE) virus and St. Louis encephalitis (SLE) virus, are endemic in certain regions of North America, emerging viruses, such as West Nile virus (WNV), have only recently been isolated in North America. The role of the black bear in the epidemiology of these viruses is not known; however, they are generally considered incidental hosts for these viruses and are not expected to serve an important role in their spread. Dunbar et al (1998) determined the seroprevalence of both alpha and flaviviruses in Florida black bears. The seroprevalence for EEE was 11 percent ( $n=66,95 \%$ CI: 4-18), Western equine encephalitis six percent $(n=66,95 \%$ CI: $0-12)$, Venezuelan equine encephalitis (VEE) three percent ( $n=11,95 \%$ CI: 0-7), and St. Louis encephalitis 17 percent ( $n=66,95 \%$ CI: 8-26) (Dunbar et al 1998). The seroprevalence of WEE $(n=334,1 \%, 95 \%$ CI: $0-2$ ) and SLE ( $n=340,1 \%, 95 \%$ CI: $0-1$ ) has also been reported for black bears captured in Idaho (Binninger et al 1980). Serologic evidence of WNV infection in black bears has been reported in New Jersey and Louisiana (Farajollahi et al 2003; Mitchell, unpublished data). The seroprevalence of WNV in black bears from New Jersey was six percent $(n=51$, 95\% CI: 0-13) (Farajollahi et al 2003), while the seroprevalence in Louisiana was 2.4 percent $(n=41$, 95\% CI: 0-6) (Mitchell, unpublished data). The role of the black bear in the transmission of arboviruses is unknown (Aguirre et al 1992). However, it is likely that bears, like humans, are dead-end hosts for these viruses. Immunocompromised individuals are more likely to be affected by arboviruses than those animals with competent immune systems.

\section{BACTERIAL Agents}

Borrelia spp. are bacterial organisms from the family Spirochaetaceae, and include B. burgdorferi, the causative agent of Lyme disease. These bacteria can cause severe disease in both humans and animals. The first isolation of Borrelia sp. from black bears was in Wisconsin (1986). Three (17\%, 95\% CI: 0-34) of the 18 bears tested for Borrelia sp. were positive (Kazmierczak et al 1988). Ixodes dammini, the primary vector of Lyme disease, was also isolated from the bears. The regions with the highest bear density in Wisconsin overlap with a known Lyme endemic area (Kazmierczak et al 1988). Because black bear numbers in Wisconsin are estimated to be low $(4,200)$, the authors of the study concluded that the black bear probably did not play an important role in the epidemiology of the disease in that state (Kazmierczak et al 1988).

Brucellosis is a zoonotic disease that can affect a number of domestic animals and wildlife. The clinical signs observed in domestic species and wildlife may include fever, diskospondylitis, abscesses, joint swelling, orchiditis, infertility, and abortion. Exposure to Brucella sp. has been reported in black bears from Idaho ( $n=332,5 \%, 95 \%$ CI: 3-7) (Binninger et al 1980), Alberta, Canada ( $\mathrm{n}=122,1 \%, 95 \%$ CI: 0-2) (Zarnke and Yuill 1981), and California ( $n=180,0.6 \%$, 95\% CI: 0-2) (Drew et al 1992), but not from Florida ( $n=37,95 \%$ CI: 0-8) (Dunbar et al 1998). In the Idaho study, it was suggested that contaminated foods (scavenging on highly infected fetuses, placentas, and/ or carcasses) and transmission to females by infected males during copulation (Binninger et al 1980) were the most likely sources of transmission. An increased seroprevalence was found in males, and the authors suggested that contaminated food was the most likely source of contamination because males have larger home ranges and an increased potential for encountering Brucella-contaminated carcasses (Zarnke and Yuill 1981). However, it is also possible that males were more likely to be seropositive because they may breed multiple females in a given year, increasing the likelihood of encountering a sexually transmitted disease. Because black bears in Idaho are predominantly her- 
bivorous on grasslands shared with domestic cattle, it has been suggested that bears might play a role in the epidemiology of brucellosis in cattle (Binninger et al 1980). The low prevalence of Brucella sp. observed in black bears from Alberta and California suggest that the bear is an unlikely reservoir for brucellosis in these areas. Any role that black bears play in the transmission and epidemiology of brucellosis is thought to be offset by the positive impact they make by removing contaminated materials from the environment (Cheville et al 1998). As clinical signs have never been reported in a black bear, it is assumed that clinical effects of brucellosis would be similar to that observed in other species, namely abortion and sterility (Zarnke 1983). Serologic surveys in the future should include testing of brucellosis, as Brucella-infected bears may be a potential health hazard to wildlife rehabilitators, hunters, researchers, and domestic animals.

Leptospira interrogans, like Borrelia sp., is a spirochete that infects domestic animals. There are over 200 different serotypes of L. interrogans. Although most of the serotypes are species specific, crossover between species does occur. This spirochete is generally spread by direct contact with infected urine or contaminated water or food sources. Wildlife rehabilitators and veterinarians working with potentially infected bears should wear exam gloves and protective eyeglasses. Leptospirosis can cause severe disease in humans and animals. The non-icteric form is less pathogenic, often causing malaise, nausea, and fever. The more severe icteric form is characterized by severe fever, blood dyscrasia, seizures, and possibly death. The seroprevalence of $L$. interrogans has been recorded in black bears from different locations. The seroprevalence of $L$. interrogans for black bears from California was 22 percent ( $n=129,95 \%$ CI: 15-29) (Ruppanner et al 1982). Leptospira interrogans serotype australis was the most likely serovar based on serologic testing (Ruppanner et al 1982). The high seroprevalence of $L$. interrogans serotype australis in these California bears was not unexpected, as rodents, the primary prey species of these bears, are the primary reservoir for that serotype (Ruppanner et al 1982). Serologic testing of black bears from Idaho ( $\mathrm{n}=196,1 \%, 95 \%$ CI: 0-2) (Binninger et al 1980) and Alaska ( $n=122,4 \%, 95 \%$ CI: 0-11) (Zarnke 1983) suggests that black bears are not routinely exposed to L. interrogans. In the Idaho and Alaska studies, L. interrogans serotype grippotyphosa, the common serotype of the raccoon, was the most common serotype in black bears. In the Alaska study, the seroprevalence increased with age, suggesting that these animals are exposed to the agent over time. As leptospirosis is considered a zoonotic disease, it is important to continually monitor the serologic status of the agent in black bears (Ruppanner et al 1982).

Campylobacteriosis and clostridial infections have also been identified in black bears. Campylobacter jejuni was isolated from the feces of one bear in Washington, although the source of infection was unknown (Pacha et al 1987). Clostridium botulinum was identified serologically in two of 123 black bears (2\%, 95\% CI: 0-4) in California (Ruppanner et al 1982). Coyotes in the same area had a seroprevalence of 23 percent, suggesting increased exposure in these pack animals (Ruppanner et al 1982). Both campylobacteriosis and clostridial infections have zoonotic potential, therefore individuals working with bears should take appropriate precautions, such as wearing exam gloves, to minimize the likelihood of contracting these bacterial pathogens. It is important to note that one case of post-immobilization clostridial myonecrosis resulted in the death of a black bear (Barnes and Rogers 1980). While the authors of this report suggested that biologists consider prophylactic antibiotics when darting black bears, the efficacy of this procedure is unknown (Barnes and Rogers 1980).

Coxiella burnetti and Rickettsia rickettsii are common rickettsial infections identified in black bears. These pathogens are primarily transmitted via ectoparasites, such as ticks, although ingestion of contaminated carcasses may also serve as a source of infection (Ruppanner et al 1982). Serologic titers to C. burnetti, the agent of Q-fever, have been found in bears from Idaho $(n=210,6 \%, 95 \%$ CI: 3-9) (Binninger et al 1980), California ( $n=149,1.7 \%, 95 \%$ CI: 0-4) (Ruppanner et al 1982) and Florida ( $n=37$, 8\%, 95\% CI: 0-17) (Dunbar et al 1998). Serologic titers to $R$. rickettsii, the cause of Rocky Mountain spotted fever, have also been found in Idaho black bears ( $n=282,2 \%, 95 \%$ CI: 0-4) (Binninger et al 1980). Seroprevalence differed with county location, and may have been associated with livestock density (Ruppanner et al 1982). Low prevalence of both rickettsial diseases suggests that the bacteria occur in relatively small amounts in black bear populations.

Francisella tularensis, the agent of tularemia, can cause severe disease in animals and humans. Clinical signs associated with this pathogen include lymphadenopathy, fever, and malaise. The transmission of this bacteria includes ectoparasites (e.g., fleas) and rodents. Black bears, like humans, are dead-end hosts for this pathogen. The seroprevalence of $F$. tularensis varies with geographic location. The incidence of tularemia is higher in the western United States than in the East. The seroprevalence of F. tularensis in black bears followed a similar pattern. Black bears from Idaho 
had a much higher seroprevalence $(n=340,19 \%$, 95\% CI: 15-23) (Binninger et al 1980) than those from Florida ( $n=40,0 \%, 95 \%$ CI: 0-7) (Dunbar et al 1998). Potential sources of the pathogen include rodents, lagomorphs, ectoparasites, and almost any species black bears prey and scavenge upon (Binninger et al 1980). In Idaho, the seroprevalence increased from 1971 to 1977 , possibly as a response to increased densities of ticks, mice, fleas, and mites (Binninger et al 1980). The authors of the study reported that black bears are capable of surviving significant exposure to F. tularensis, evident by increased titers discovered upon recapture (Binninger et al 1980). However, this has not been determined experimentally.

Serologic titers to Yersinia pestis, the agent of plague, have only been identified in California black bears. The seroprevalence varied with location, ranging from 15 percent to 36 percent (Ruppanner et al 1982; Smith et al 1984; Clover et al 1989). Exposure in these animals was most likely associated with ectoparasites (rodent fleas) or through the ingestion of infected rodents, the primary reservoir of plague (Ruppanner et al 1982). Transmission through ingestion of infected rodents is more probable because viable Y. pestis could not be isolated from any flea pools, and rodent hairs were found in six percent of the bear fecal samples (Clover et al 1989). Small mammals comprised less than one percent of the black bear diet in Tahoe National Forest in California; however, this may vary with season (Clover et al 1989). No variation in seroprevalence was found for sex, age, or year of capture. Antibody titers have been demonstrated to persist between two to seven months (Clover et al 1989), suggesting that the black bear is not a reservoir for the agent (not persistently infected). Serologic evidence of exposure in rodent-eating animals, such as the black bear, can provide an early warning for the spread of Y. pestis that would otherwise require repeated rodent surveys.

\section{CONCLUSION}

Black bears are a keystone species in North America. Because of the omnivorous feeding strategy used by these animals, exposure to infectious diseases might be expected. Black bears can also serve as important sentinels of infectious disease in an ecosystem. Wildlife rehabilitators, biologists, and veterinarians working with black bears should be aware of potential infectious diseases and take appropriate precautions to minimize the likelihood of zoonotic disease transmission.

\section{LITERATURE CITED}

Aguirre, A. A., R. G. McLean, et al. 1992. Serologic survey for selected arboviruses and other potential pathogens in wildlife from Mexico. J Wildlife Diseases, 28(3):435-442.

Barnes, D. M., and L. L. Rogers. 1980. Clostridial myonecrosis in a black bear associated with drug administration. J Wildlife Diseases, 16(3):315-317.

Binninger, C. E., J. J. Beecham, et al. 1980. A serologic survey for selected infectious diseases of black bears in Idaho. J Wildlife Diseases, 16(3):423-430.

Cheville, N. F., D. R. McCullough, and L. R. Paulson. 1998. Brucellosis in the Greater Yellowstone area. National Research Council. Washington, DC: National Academy Press.

Clover, J. R., T. D. Hofstra, et al. 1989. Serologic evidence of Yersinia pestis infection in small mammals and bears from a temperate rainforest of north coastal California. J Wildlife Diseases, 25(1):52-60.

Collins, J. E., P. Leslie, et al. 1984. Epizootic of adenovirus infection in American black bears. Journal of the American Veterinary Medical Association, 185(11):1430-1432.

Drew, M. L., D. A. Jessup, et al. 1992. Serologic survey for brucellosis in feral swine, wild ruminants, and black bear of California, 1977 to 1989. J Wildlife Diseases, 28(3):355-363.

Dunbar, M. R., M. W. Cunningham, et al. 1998. Seroprevalence of selected disease agents from free-ranging black bears in Florida. J Wildlife Diseases, 34(3):612-619.

Farajollahi, F., N. A. Panella, et al. 2003. Serologic evidence of West Nile virus infection in black bears (Ursus americanus) from New Jersey. J Wildlife Diseases, 39(4):894-896.

Hassard, T. H. 1991. Estimation. In Understanding Biostatistics. T. H. Hassard (ed). St. Louis, MO: Mosby Year Book. Pp. 38-51.

Kazmierczak, J. J., T. E. Amundson, et al. 1988. Borreliosis in free-ranging black bears from Wisconsin. J Wildlife Diseases, 24(2):366-368.

Pacha, R. E., G. W. Clark, et al. 1987. Small rodents and other mammals associated with mountain meadows as reservoirs of Giardia spp. and Campylobacter spp. Applied and Environmental Microbiology, 53(7):1574-1579.

Pirtle, E. C., M. E. Roelke, et al. 1986. Antibodies against pseudorabies virus in the serum of a Florida black bear cub. Journal of the American Veterinary Medical Association, 189(9):1164.

Pursell, A. R., B. P. Stuart, et al. 1983. Isolation of an adenovirus from black bear cubs. J Wildlife Diseases, 19(3):269-271. 
Ruppanner, R., D. A. Jessup, et al. 1982. Serologic survey for certain zoonotic diseases in black bears in California. Journal of the American Veterinary Medical Association, 181(11):1288-1291.

Schultze, A. E., R. K. Maes, et al. 1986. Pseudorabies and volvulus in a black bear. Journal of the American Veterinary Medical Association, 189(9):1165-1166.

Smith, C. R., B. C. Nelson, et al. 1984. The use of wild carnivore serology in determining patterns of plague activity in rodents in California. Proceedings Eleventh Vertebrate Pest Conference. Pp. 71-76.

van Belle, G., and S. P. Millard. 1998. STRUTS:

Statistical Rules Of Thumb. <www.nrcse.washington. edu>. Seattle, WA. Pp. 3-14.

van der Leek, M. L., H. N. Becker, et al. 1993.

Prevalence of pseudorabies (Aujeszky's disease) virus antibodies in feral swine in Florida. $J$ Wildlife Diseases, 29(3):403-409.

Walroth, R., N. Brown, et al. 1996. Rabid black bears in Ontario. Canadian Veterinary Journal, 37(8):492.

Wandeler, A. I., and G. A. Casey. 1993. Eastern Canada rabies diagnoses, January 1-June 30, 1992. Canadian Veterinary Journal, 34:123.

Whetstone, C. A., H. Draayer, et al. 1988.

Characterization of canine adenovirus type 1 isolated from American black bears. American Journal of Veterinary Research, 49(6):778-780.

Zarnke, R. L., and T. M. Yuill. 1981. Serologic survey for selected microbial agents in mammals from Alberta, 1976. J Wildlife Diseases, 17(3):453-461.

Zarnke, R. L. 1983. Serologic survey for selected microbial pathogens in Alaskan wildlife. J Wildlife Diseases, 19(4):324-329. (․ㅏㅅ

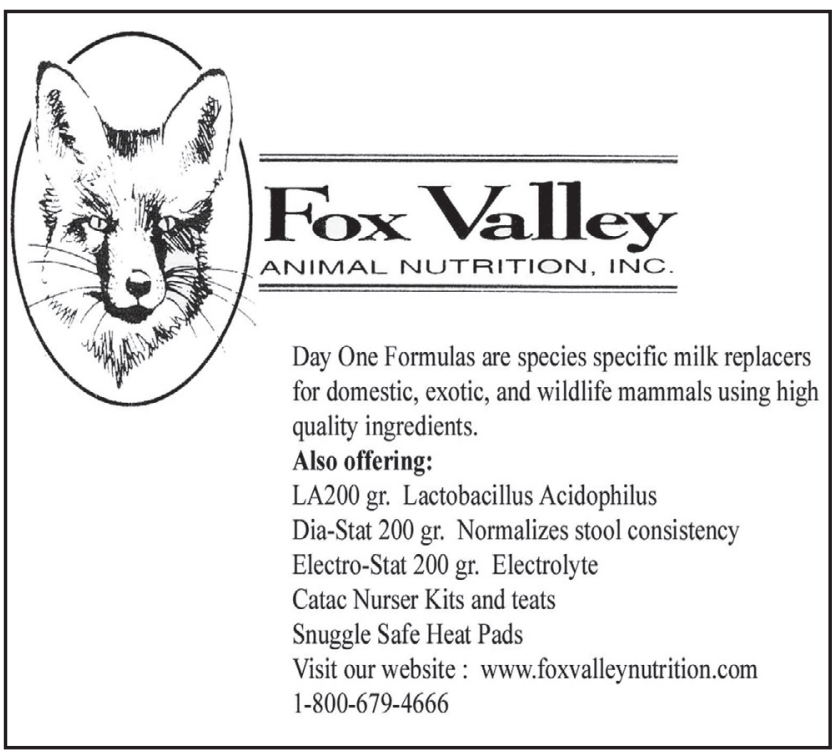

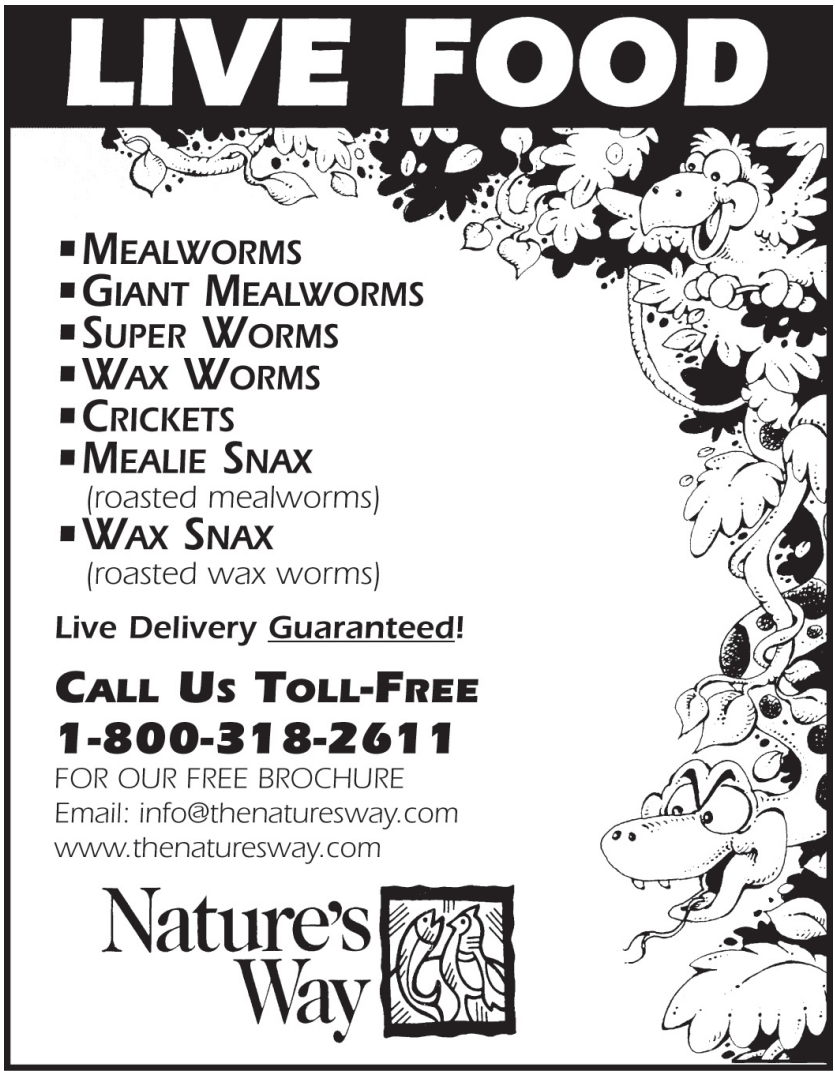

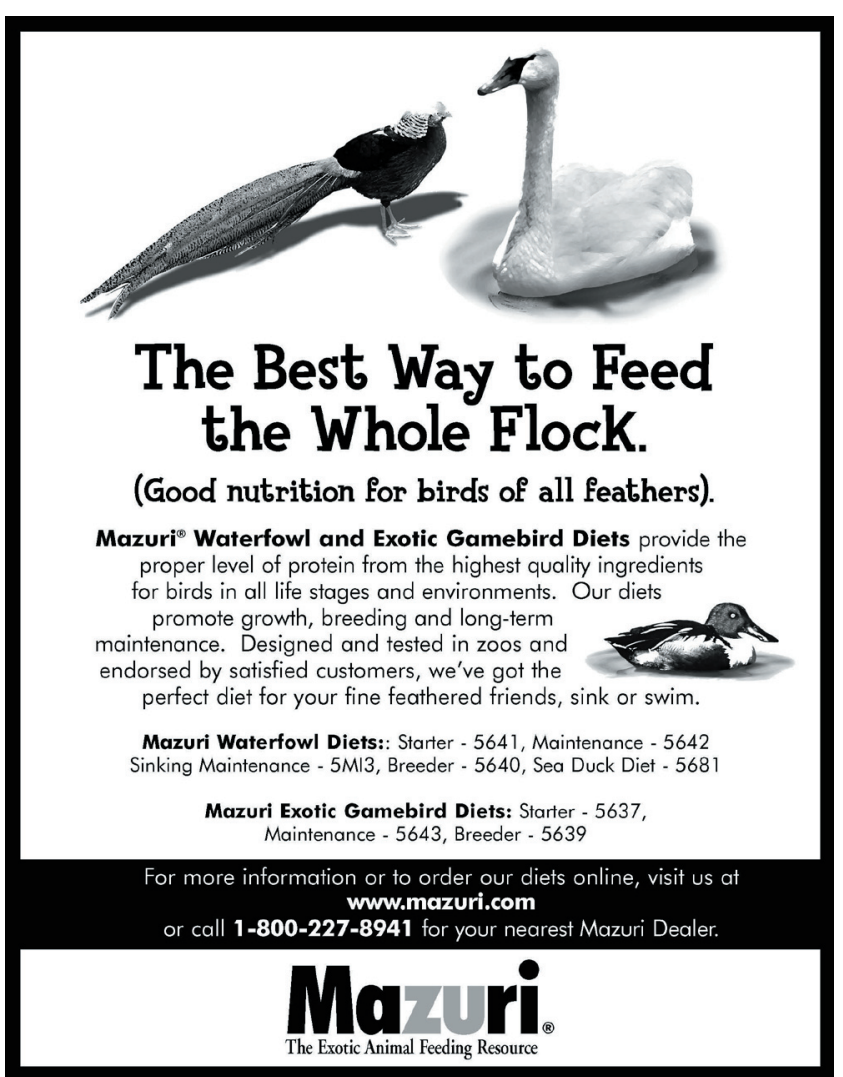

